\title{
VIII. Notes sur la flore espagnole et portugaise. Troisième voyage en Portugal
}

(Suite et fin);

PAR M. MICHEL GANDOGER.

Quelques jours après, nos récoltes mises en ordre, nous repartîmes pour une autre sierra tout aussi éloignée et tout aussi inconnue, la cordillère de Francia. C'est l'une des régions les plus tourmentées et les plus bizarres que j'aie vues en Espagne. Blasé sur les paysages, je fus vraiment impressionné à la vue de ce chaos de cònes, de pics, de précipices qui avoisinent la Peña, point le plus élevé de la chaîne. Cette expédition, des plus pénibles, nous prit presque une semaine. Mais que de découvertes vinrent nous dédommager! Je cite :

Aquilegia hispanica (Willk.)

Ranunculus Aleæ

- Hollianus

- Lagascanus (Noureau).

Alyssum atlanticum (Nouveau pour l'Espagne centrale).

Brassica valentina

Lepidium calycotrichum

Cistus hirsutus, etc.

Helianthemum cheiranthoides, etc.

Arenaria capitata

- erinacea (Nouveau).

Cerastium Riæi

Dianthus hispanicus

- Langeanus

- laricifolius

Genista triacanthos

- Tournefortii

- lasiantha

Conopodium Bourgæi

Physospermum aquilegifolium

Thapsia transtagana

Galium Broterianum

Evax exigua
Evax carpetana

Pyrethrum flaveolum (Tres rare). Jasione carpetana (Nouieau).

Erica aragonensis, etc.

Fraxinus angustifolia

Omphalodes nitida

Linaria nivea

Armeria scorzonerifolia

Betula pubescens (Nouveau pour l'Espagne centrale).

Quercus Tozza et lusitanica, avec de nombreuses formes.

Allium stramineum (Nouveau).

Simethis bicolor

Asphodelus microcarpus, etc.

Agrostis hispanica

Kœleria crassipes

Molineria minuta

Nardurus patens Hack. (Nouveau pour l'Espagne).

Trisetum ovatum

Cystopteris montana

Blechnum Spicant (Nouveau pour la province).

La province de Léon, encore peu connue, m'attirait pour la seconde fois. Fixé à Astorga, j'y récoltai 137 numéros dont je

1. Voir plus haut, p. 104. 
I. GANdOger. - NOTES SUR LA FlokE kSPAgNole ET pORTUgaise. 133

ne puis guère citer que : Sisymbrium contortum, Trifolium gemellum, Peplis longedentata, Senecio foliosus, Hypocheris adscendens, Convolvulus Cherleri, Elymus Caput-Medusx, Molineria minuta et surtout le rarissime Genista ancistrocarpa Spach, nouveau pour l'Espagne et absent de la plupart des grands herbiers, ne l'ayant pas moi-mème.

Une autre plante extrèmement rare et manquant dans toutes les collections était le Senecio legionensis Lge, cantonné dans une prairie au Puerto de Manzanal (Léon) et que personne n'avait récoltée depuis LANGE, c'est-à-dire depuis plus de quarante ans. J'allai donc à ladite localité et je descendis à la station de Brañuelas qui en est la plus rapprochée. Je sortais de la gare lorsque mon domestique français me fit remarquer, à droite de la voie, une prairie d'aspect jaunâtre. Nous y allàmes et des centaines de pieds du fameux Senecio en parfait état n'attendaient qu'une heureuse main qui pùt les centurier et les distribuer. C’est ce que j’ai fait. Sur mon désir, M. Dörfler, de Vienne, a coté cette plante 1300 unités (9 francs l'exemplaire) seulement, bien qu'elle vaille le triple.

Nous herborisàmes non seulement à Brañuelas, mais aussi au Puerto de Manzanal où j’ai revu ce précieux Senecio legionensis. C'est une excursion que je recommande vivement, car les deux localités sont des plus riches du nord de l'Espagne qui en compte cependant bien d'autres.

Parmi les 221 numéros récoltés je mentionnerai :

Batrachium tripartitum

Ranunculus pseudo-reptans

Cheiranthus linifolius

Helianthemum occidentale

- Langei

Dianthus Planella

Malva Colmeiroi (Très rare).

Genista cantabrica

- stenoptera

- leptoclada

- polygalifolia

- micrantha

- Jusitanica

Sedum brevifolium

Geocaryum capillifolium

Galium Halleri

- Helodes
Carduus Gayanus

Centaurea carpetana

Pyrethrum pulverulentum

Senecio legionensis Lange!

- foliosus $f$. Duriæi Nym.

Calluna vulgaris $f$. tomentosa, an species nova?

Erica Martinesii (Noureau).

- umbellata

- aragonensis

Myosotis stolonifera var.

Veronica parmularia

Armeria Duriæi

Plantago acanthophylla

Allium stramineum (Nouveau pour le nord de l'Espagne).

Ornithogalum unifolium 
Romulea grandiflora?

Damasonium Bourgæi (Nouveau). - minimum Lge (Très rare!)

Luzula lactea

Aira montana

Avena sulcata

Kœleria crassipes

Periballia hispanica

Nardurus patens (Novveau pour le Nord de l'Espagne).

Potamogeton polygonifolius

Nombreuses Mousses et Lichens.

En me rendant dans les Asturies de la province de Palencia et de Santander, je passais au pied du Puerto de Pajarès et du mont Arvas que j'avais explorés en 1898 et où j'avais trouvé tant de raretés et de nouveautés. J'y fis une seconde excursion; j'y trouvai, au 24 juin, la végétation dans toute sa splendeur jusqu'aux neiges, à 2200 mètres, les bestiaux n'ayant heureusement pas encore pénétré dans cette exceptionnelle région. Sur 246 plantes je n'indique que les suivantes pour ne pas me répéter :

Aquilegia discolor

Caltha minor

Ranunculus amplexicaulis

Cardamine latifolia

Iberis conferta

Erucastrum subbipinnatifidum

Lepidium arvasense

- Smithii

Viola cæspitosa $f$. asturica

Dianthus Langeanus

Sagina sabuletorum

Silene arvatica

Spergula viscosa

Genista obtusiramea

- leptoclada

- cantabrica

Sarothamnus cantabricus

- commutatus

Saxifraga trifurcata

- umbrosa

Angelica lævis

Conopodium subcarneum

Eryngium Bourgati

Levisticum officinale. Busdongo.

Carduncellus monspeliensium

Carduus arctioides Willd. (Nouveau pour l'Espagne).
Jurinea pyrenaica

Phalacrocarpum oppositifolium

Adenostyles pyrenaica

Doronicum carpetanum

Senecio foliosus $f$. asturica

Solidago alpestris

Crepis lampsanifolia

Hieracium legionense

Campanula acutangula

Jasione genisticola

- carpetana $f$. asturica

Dabœcia polifolia

Ajuga pyramidalis var.

Thymus nervosus

Erinus glabratus

Linaria pyrenaica

Pedicularis verticillata (Très rare en Espagne).

Veronica Tournefortii

Plantago incana

Rumex suffruticosus

Daphne cantabrica

Thymelæa coridifolia

Luzula cæspitosa

Carex asturica

- Cantabrorum sp. n. ${ }^{1}$.

Apera interrupta

1. Carex cantabrorum Gdgr. mss. Affinis C. rigidæ Good. in Hispania hucusque non lectæ, a qua certe differt cæspitibus multo majoribus, copiose ac longe surculosis, foliis concoloribus, margine vix aspero-serru- 
M. GaNdOger. - NOTES SUR LA Floke ESPAgnole et portugaise. 135

Brachypodium rupestre

Fèstuca Eskia

Kœleria setacea

Avena sulcata

Poa alpina, avec une variété voisine du P. cenisia et qui serait peutêtre le P. commutata R. S. qui n'a pas été retrouvé dans son unique localité près du mont Arvas depuis un siècle.

Un massif montagneux inexploré est celui de la Peña Corada (prov. de Léon). On s'établit à Cistierna. Le pays est calcaire, avec végétation très variée. Entre 1000 et 1833 mètres j’ai récolté 36 numéros. En voici quelques-uns :

Helleborus viridis var.

Hutchinsia Auerswaldii

Isatis canescens

Alsine palentiana

- laricifolia

Cerastium Riæi

Dianthus Langeanus

Silene legionensis

Malva geranifolia

- Tournefortiana

Adenocarpus parvifolius

Anthyllis alpestris

Astragalus macrorrhizus

Genista polygalifolia

Alchemilla hybrida

Geum sylvaticum

Rosa, 19 espèces

Spiræa Cavanillesii

Paronychia aretioides

Pistorinia hispanica

Sedum atratum (Nourealı pour la province.

Saxifraga castellana

- conifera (Nouveau pour la prov.)
Conopodium Bourgai $f$. pumila

Daucus Bertolonii

Physospermum cornubiense (Nouveau).

Pimpinella siifolia (Noureau).

Galium tenellum

- aciphyllum

Valeriana tuberosa

Globularia nana

Centaurea lingulata

- castellana $f$. lanuginosa

Cirsium castellanum

Achillea microphylla $f$. tomentosa

Evax exigua

Filago prostrata

Santolina canescens

Crepis pulchra

Campanula hispanica

Jasione sessiliflora

- carpetana (Nouveau).

Erica aragonensis

Anagallis linifolia

Brunella hyssopifolia

Mentha candicans

latis, elongatis, spicæ masculæ squamis retusis, fimbriatis vel incisis, latissime scariosis, spicæ femineæ vero obovatis, cuspidatis, elevatim carinatis, ferrugineis, anguste marginatis, capsula duplo majore, oblonga, utrinque attenuata, costata, obtuse trigona magisque stylosa, squamis subduplo longiore. Junio-Julio.

Hab. Astur., in cacumine montis Arvas ad nives cum C. asturica promiscue crescens, alt. $2100 \mathrm{~m}$.; in monte Pozo de Curavacas versus lacunas superiores, alt. $2330 \mathrm{~m}$.; in monte Peña Labra (Palencia) copiose in declivibus siccis, alt. 17-1900 m. et probabilius alibi his in montanis additu difficillimis, quos futuris junioribusque botanicis enixe commendo.

Planta nunc a cl. H. LÉvelLlé ad $C$. paniceam L., nunc a meipso sive ad hanc, sive ad. C. asturicam B. R., sive tandem ad C. intricatam Tineo passim relata, certissime ad nullam harum pertinet, ac speciem novam, habitu peculiari, prope $C$. rigidam Good. collocandam, constituit. 
Thymus serpylloides (Nouveau pour le Nord de l'Espagne).

Digitalis parviflora

Orobanche Spartii

Plantago capitellata

Rumex pyrenaicus

Quercus lusitanica

Taxus baccata

Narcissus biflorus

Merendera Bulbocodium

Luzula lactea

Agrostis Duriæi

Festuca Hystrix

- plicata
Hordeum leporinum

Kœleria crassipes

Phleum microstachyum

Poa ligulata

Oreochloa pallida Gdgr Fl. Eur. xxvı, p. 238 (1891). O. confusa De Coincy (1896). - Cette espèce est assez répandue dans les hautes montagnes calcaires des provinces de Léon, de Santander et de Palencia. En 1908 je l'ai retrouvée dans la sierra de la Demanda (Logroño).

Ces montagnes calcaires de la chaìne cantabrique ont une végétation extraordinairement variée. Témoin encore le mont Espiguëte, qui atteint 2450 mètres. Nous y récoltàmes 416 plantes. C'est une herborisation hors ligne qui peut se comparer avec les plus fructueuses à faire en Espagne, sierras Tejeda, de Mijas, de Yunquera, etc., en Andalousie. - Je mentionne surtout :

Aconitum Lycoctonum

Ranunculus carpetanus

- nodiflorus (Nouveau).

Arabis cantabrica (Nouveau).

Erucastrum subbipinnatifidum

Brassica montana

Erysimum Bourgæanum (Nouveau pour le nord de l'Espagne).

Sisymbrium asperum

- contortum

Helianthemum marifolium

- cheiranthoides

Agrostemma gracilis Boiss. (Nouveau pour l'Espagne).

Alsine palentiana (Nouveau .

Arenaria cantabrica

Silene legionensis

Malva geranifolia

Rhamnus alpinus

Erodium petræum (Nouveau).

Anthyllis Webbiana

Astragabus nevadensis

- depressus (Nouveau).

Genista lasiantha

- cantabrica

- micrantha
Genista leptoclada

- polygalifolia $f$. microphylla

- obtusiramea

Sarothamnus cantabricus

- commutatus

- Bourgæi (Nouveau).

Rosa resinosa

Herniaria latifolia

Sedum pyrenaicum

Saxifraga canaliculata

- conifera (Nouveau).

Conopodium subcarneum

Endressia pyrenaica (Nouveau pour

la chaîne cantabrique).

Laserpitium pubescens

Lugusticum pyrenæum

Centaurea Grællsii (Nouveau).

Cirsium filipendulum

Gnaphalium carpetanum (Nouveau).

Senecio Duriæi

Hieracium bombycinum

- balsameum

Omalocline granatensis (Nouveau). Jasione sessiliflora, etc.

Arctostaphylos Uva-ursi

Verbascum virgatum 
M. GaNd0ger. - NOTES SUR LA Flore ESPAgNole ET PORTUgaise. 137

Anchusa sempervirens

Cynoglossum clandestinum

- nebrodense

Nepeta tuberosa (Nouveau pour le Nord de l'Espagne).

Sideritis hirtula

Teucrium pyrenaicum

Digitalis parviflora

Euphrasia Willkommii (Nouveau).

Linaria delphinioides

- filicaulis (Nouveau).

- faucicola (Nouveau; rarissime).

Scrofularia oblongifolia

Armeria castellana (Nouveau).
Daphne cantabrica

Salix versifolia? vel sp. nova?

Corbularia cantabrica

Juniperus Sabina

Agrostis Langei

Aira flexuosa $f$. brachyphylla

Avena cantabrica

Festuca hystrix

Oreochloa pallida $f$. asturica

Poa ligulata

Trisetum ovatum

- purpurascens (Noureau).

- hispidum Lge. Espèce pcu connue. Abonde dans la région.

Une autre chaìne, la sierra del Brezo (1 959 mètres), voisine de l'Espiguëte, est également des plus intéressantes. J'y ai récolté 356 numéros, parmi lesquels je cite :

Conringia orientalis

Erysimum Bourgæanum

Helianthemum eriocaulon, etc.

Alsine Villarsii

Arenaria modesta

Dianthus lusitanus

- Langeanus

Linum salsoloides

Adenocarpus parvifolius

Coronilla Clusii

Hippocrepis prostrata

Onobrychis Reuteri (Nouveau).

Ononis cenisia

Orobus pannonicus

Trifolium cerverense (Nouveau).

Vicia lancifolia

Rosa hispanica, etc.

Spiræa Cavanillesii

Saxifraga conifera

- canaliculata $f$. compacta

Eryngium pusillum

Pastinaca hirsuta (Nouveau).

Thapsia minor

Galium Helodes

- anisophyllum
Knautia subscaposa (Noureau).

Carduus Gayanus

Centaurea carpetana

- cephalarifolia

- Grællsii (Nouveau).

Cirsium crinitum

- castellanum

Serratula pinnatifida

Artemisia Assoana

Senecio minutus, etc.

Barkhausia macrocephala

Scorzonera pinifolia

Erica aragonensis, etc.

Nepeta Nepetella

Thymus algeriensis (Noureau).

Linaria pyrenaica

Armeria castellana

Plantago capitellata

- discolor (Nouveau).

Quercus lusitanica $\times$ Tozza sp. n. ${ }^{1}$.

Salix ambigua

- oleifolia

- pentandra (Nouveau).

Agropyrum caninum (Nouveau).

Brachypodium rupestre

1. Quercus lusitanica $\times$ Tozza Gdgr mss. Folia ut in $Q$. lusitanica sed majora subtusque villosa, acute dentata; iuflorescentia Q. Tozzam refert. Ab utroque statura humili, ramis laxis, foliis supra opacis, etc., recedit. - Aliquot exstant arbores prope Villaverde (Palencia) ad pedem s. del Brezo, alt. $1100 \mathrm{~m}$. 
Elymus Caput-Medusæ

Festuca sulcata

Oreochloa pallida

Phleum microstachyum
Poa firmula

Ventenata avenacea (Nouveau pour le Nord de l'Espagne).

Cystopteris regia.

En terminant je citerai quelques-ünes des plantes récoltées autour de Badajoz lors de mon séjour à Elvas, sur la frontière portugaise :

Brassica lævigata

Silene apetala

Althæa rosea

Lavatera sylvestris

Malva hispanica

Hypericum tomentosum

Astragalus hypoglottis

Eryngium galioides

Galium fruticescens

Onopordum nervosum

Cichorium pumilum

Picridium intermedium

Fraxinus angustifolia

Anagallis linifolia

Chlora imperfoliata
Convolvulus Cherleri
Nepeta tuberosa
Salvia lanigera
- argentea
Thymus Zygis
Scrofularia frutescens
Mercurialis tomentosa var.
Celtis australis
Brachypodium pentastachyum
Hordeum maritimum
Trisetum neglectum
Stipa parviflora

Chlora imperfoliata Convolvulus Cherleri Nepeta tuberosa Salvia lanigera

- argentea

Thymus Zygis

Scrofularia frutescens

Mercurialis tomentosa var.

Celtis australis

Brachypodium pentastachyum

Hordeum maritimum

Trisetum neglectum

I

Enfin Colmeiroa buxifolia Reut. sur les bords de la Guadiana, arbuste rare dans la Péninsule et manquant dans beaucoup d'herbiers, si j'en juge par les demandes qu'on m'adresse fréquemment.

M. Guillaumin fait la communication suivante :

\section{Observations sur les Burséracées de Madagascar;}

par M. A. Guillaumin.

Bien que l'inventaire des richesses florales de Madagascar soit encore loin d'être terminé et que certains points de notre colonie soient encore presque inconnus, nos connaissances sur la flore malgache commencent à se préciser. Les précieuses observations et les collections recueillies depuis un siècle par Chapelier, Boivin, Grévé, Pervillé, Perrier de la Bathie, Grandidier, Baker, Barox, publiées par ces deux derniers auteurs dans le Journal de la Société Linnéenne de Londres et la Revue de Madagascar, et par Baillon dans la belle Monographie de Madagascar par M. Grandidien, nous permettent de dire que les Burséracées, si 


\section{$2 \mathrm{BHL}$ Biodiversity Heritage Library}

Gandoger, Michel. 1909. "VIII. Notes sur la flore espagnole et portugaise. Troisième voyage en Portugal." Bulletin de la Société botanique de France 56, 132-138. https://doi.org/10.1080/00378941.1909.10831381.

View This Item Online: https://www.biodiversitylibrary.org/item/8678

DOI: https://doi.org/10.1080/00378941.1909.10831381

Permalink: https://www.biodiversitylibrary.org/partpdf/160783

\section{Holding Institution}

Missouri Botanical Garden, Peter H. Raven Library

\section{Sponsored by}

Missouri Botanical Garden

\section{Copyright \& Reuse}

Copyright Status: Public domain. The BHL considers that this work is no longer under copyright protection.

This document was created from content at the Biodiversity Heritage Library, the world's largest open access digital library for biodiversity literature and archives. Visit BHL at https://www.biodiversitylibrary.org. 